\title{
Surface Compression With Hierarchical Powell-Sabin B-Splines
}

\author{
Jan Maes Adhemar Bultheel
}

Report TW 391, May 2004

\section{Katholieke Universiteit Leuven}

\section{Department of Computer Science}

Celestijnenlaan 200A - B-3001 Heverlee (Belgium) 


\title{
Surface Compression With Hierarchical Powell-Sabin B-Splines
}

\author{
Jan Maes \\ Adhemar Bultheel
}

Report TW 391, May 2004

Department of Computer Science, K.U.Leuven

\begin{abstract}
We show how to construct a stable hierarchical basis for piecewise quadratic $C^{1}$ continuous splines defined on Powell-Sabin triangulations. We prove that this hierarchical basis is well suited for compressing surfaces. Our compression method does not require the construction of wavelets which are usually expensive to compute, but instead we construct a stable quasi-interpolation scheme for our spline space which achieves optimal approximation order. Numerical experiments demonstrate the high compression rate of the algorithm.
\end{abstract}

Keywords : Powell-Sabin Splines, Hierarchical Bases, Surface Compression, Stable Approximation by Splines

AMS(MOS) Classification : 41A15, 65D15, 65D17 


\title{
Surface Compression With Hierarchical Powell-Sabin B-Splines
}

Jan Maes

\author{
Adhemar Bultheel
}

May 2004

\begin{abstract}
We show how to construct a stable hierarchical basis for piecewise quadratic $C^{1}$ continuous splines defined on Powell-Sabin triangulations. We prove that this hierarchical basis is well suited for compressing surfaces. Our compression method does not require the construction of wavelets which are usually expensive to compute, but instead we construct a stable quasi-interpolation scheme for our spline space which achieves optimal approximation order. Numerical experiments demonstrate the high compression rate of the algorithm.
\end{abstract}

\section{Introduction}

In this paper we look for a compression algorithm for the space $S_{2}^{1}\left(\Delta_{P S}\right)$ of piecewise quadratic $C^{1}$ continuous splines on Powell-Sabin (PS) triangulations which does not require the construction of wavelets. Instead we use a hierarchical basis. Hereto we were inspired by the work of Hong and Schumaker [5] who have constructed a surface compression algorithm for the space of $C^{1}$ cubic splines defined on triangulations obtained from convex quadrangulations. Although stable wavelets for the space $S_{2}^{1}\left(\Delta_{P S}\right)$ have been constructed in [11] we prefer not to use these wavelets for surface compression because of their substantial amount of computation time. The method presented here using hierarchical bases is easy to implement and is computationally efficient. We do not need to solve any systems of equations in either the decomposition or reconstruction phases.

Let $\Omega$ be a bounded polygonal domain in $\mathbb{R}^{2}$. The key to our compression method is the construction of a linear quasi-interpolation operator $Q$ which maps $C(\Omega)$ onto the spline space $S_{2}^{1}\left(\Delta_{P S}\right)$ in such a way that if $f$ lies in a Sobolev space $W_{p}^{k+1}(\Omega)$ with $1 \leq k \leq 2$ and $2 \leq p \leq \infty$, then $Q f$ approximates $f$ and its derivatives to optimal order,

$$
\left\|D_{x}^{\alpha} D_{y}^{\beta}(f-Q f)\right\|_{p, \Omega} \leq C|\Delta|^{k+1-\alpha-\beta}|f|_{k+1, p, \Omega}, \quad 0 \leq \alpha+\beta \leq k+1 .
$$

Here $|\Delta|$ is the diameter of the largest triangle in the triangulation $\Delta$ of the domain $\Omega$. In a hierarchical context $|\Delta|$ will become smaller and smaller with each resolution level. We will exploit this fact to prove that a lot of coefficients in a hierarchical surface representation can be neglected which yields the surface compression.

The outline of the paper is as follows. Section 2 is devoted to the idea of hierarchical bases and we discuss their usefulness for compression purposes. In Section 3 we recall some general concepts of polynomials on triangles and we review the relevant aspects and properties of a normalized B-spline basis for Powell-Sabin splines. In Section 4 we show how to construct a stable quasi-interpolation scheme for our normalized B-spline basis which achieves optimal approximation order. Sections 5 and 6 are devoted to the construction of a hierarchical basis, while Section 7 goes into the details of the compression algorithm. Section 8 demonstrates the compression algorithm with some numerical examples, and we conclude the paper with an application to image compression. 


\section{Hierarchical Bases}

The starting point for the idea of hierarchical bases is a nested sequence of finite dimensional spaces of real-valued functions

$$
V^{0} \subset V^{1} \subset V^{2} \subset \ldots \subset V^{l} .
$$

As $j$ increases, the resolution of functions in $V^{j}$ increases. Each space $V^{j}$ has a finite basis and a set of functions

$$
\mathcal{B}:=\bigcup_{j=0}^{l}\left\{\mathcal{B}_{i}^{j}\right\}_{i=1}^{n_{j}}
$$

is a hierarchical basis for $V^{l}$ given that

$$
\mathcal{B}^{m}:=\bigcup_{j=0}^{m}\left\{\mathcal{B}_{i}^{j}\right\}_{i=1}^{n_{j}}
$$

is a basis for $V^{m}$ for each $m=0,1, \ldots, l$. Then every $s \in V^{l}$ can be written in the form

$$
s=\sum_{j=0}^{l} \sum_{i=1}^{n_{j}} c_{i}^{j} \mathcal{B}_{i}^{j},
$$

and the partial sums

$$
s_{m}=\sum_{j=0}^{m} \sum_{i=1}^{n_{j}} c_{i}^{j} \mathcal{B}_{i}^{j},
$$

are functions in the spaces $V^{m}$ for each $m=0,1, \ldots, l$. The hierarchical expansion (2.3) is especially useful when the partial sums $s_{0}, s_{1}, \ldots, s_{l-1}$ can be regarded as better and better approximations of $s$, i.e.

$$
\left\|s-s_{0}\right\|>\left\|s-s_{1}\right\|>\ldots>\left\|s-s_{l-1}\right\| .
$$

Now we can approximate $s$ at different levels of detail by eliminating the "finer" coefficients. For example, the coarsest approximation $s_{0}$ only uses the coefficients $\left\{c_{i}^{0}\right\}_{i=1}^{n_{0}}$.

Suppose that for all $m$ the basis functions $\mathcal{B}^{m}$ form a stable basis for $V^{m}$, i.e. that there exist constants $k_{1}$ and $k_{2}$ such that for all choices of the coefficient vector $\mathbf{c}^{m}$

$$
k_{1}\left\|\mathbf{c}^{m}\right\| \leq\left\|\mathcal{B}^{m} \mathbf{c}^{m}\right\| \leq k_{2}\left\|\mathbf{c}^{m}\right\|,
$$

then (2.5) expresses that small changes in the size of the coefficients in (2.3) lead to small changes in the size of $\|s\|$. Hence the expansion (2.3) can be used for compression.

\section{Powell-Sabin splines}

We are interested in the spline space $S_{2}^{1}\left(\Delta_{P S}\right)$, that is the space of piecewise quadratic $C^{1}$ continuous functions on a Powell-Sabin refinement $\Delta_{P S}$. In this section we briefly review the B-spline representation, the geometric interpretation with control triangles and the stability of the basis.

Consider a triangle $\mathcal{T}\left(V_{1}, V_{2}, V_{3}\right)$ in a plane with vertices $V_{i}, i=1,2,3$. Define $\mathcal{P}_{2}$ as the space of bivariate polynomials of total degree $\leq 2$, then each polynomial $P_{2}(x, y) \in \mathcal{P}_{2}$ on $\mathcal{T}$ has a unique representation

$$
P_{2}(x, y)=\sum_{|\lambda|=2} b_{\lambda} B_{\lambda}^{2}(\tau)
$$

with $\lambda=\left(\lambda_{1}, \lambda_{2}, \lambda_{3}\right), \quad \lambda_{i} \geq 0$ a multi-index of length $|\lambda|=\lambda_{1}+\lambda_{2}+\lambda_{3}=2, \tau=\left(\tau_{1}, \tau_{2}, \tau_{3}\right)$ the barycentric coordinates of $(x, y)$ with respect to $\mathcal{T}$, and

$$
B_{\lambda}^{2}(\tau)=\frac{2 !}{\lambda_{1} ! \lambda_{2} ! \lambda_{3} !} \tau_{1}^{\lambda_{1}} \tau_{2}^{\lambda_{2}} \tau_{3}^{\lambda_{3}}
$$




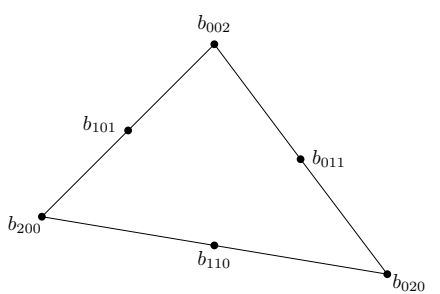

Figure 1: Positions of the Bézier ordinates.

the Bernstein-Bézier polynomials of degree 2 on the triangle [4]. The coefficients $b_{\lambda}$ are called the Bézier ordinates. The points $\left(\frac{\lambda}{2}, b_{\lambda}\right)$ are the control points for the surface $z=P_{2}(x, y)$ and the piecewise linear interpolant to these points is the Bézier net or control net. We can display this Bernstein-Bézier representation schematically, as in Figure 1. The points $\frac{\lambda}{2}$, marked with dots on the figure, are called the Bézier domain points.

Consider a simply connected subset $\Omega \subset \mathbb{R}^{2}$ with polygonal boundary $\delta \Omega$. Suppose we have a conforming triangulation $\Delta$ of $\Omega$, consisting of triangles $\mathcal{T}_{j}, j=1, \ldots, t$, and having vertices $V_{i}$ with Cartesian coordinates $\left(x_{i}, y_{i}\right), i=1, \ldots, n$. The Powell-Sabin refinement $\Delta_{P S}$ of $\Delta$ divides each triangle $\mathcal{T}_{j}$ into six subtriangles with a common vertex. It can be constructed as follows (see Figure 2a):

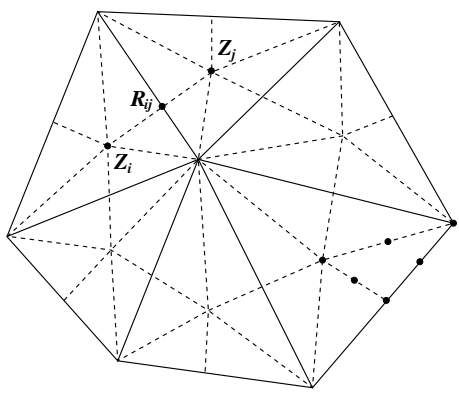

(a) a PS-refinement $\Delta_{P S}$

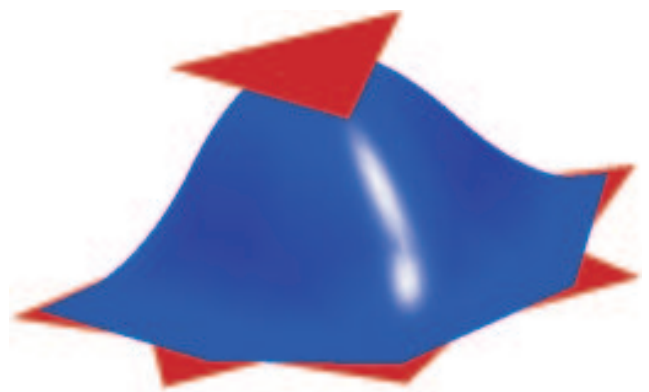

(b) a PS B-spline surface with control triangles

Figure 2: B-spline representation for $S_{2}^{1}\left(\Delta_{P S}\right)$.

1. Choose an interior point $Z_{j}$ for each triangle $\mathcal{T}_{j}$, so that if two triangles $\mathcal{T}_{i}$ and $\mathcal{T}_{j}$ have a common edge, the line joining $Z_{i}$ and $Z_{j}$ intersects this common edge at a point $R_{i j}$ between its vertices. We will choose $Z_{j}$ as the incenter of triangle $\mathcal{T}_{j}$.

2. Join the points $Z_{j}$ to the vertices of $\mathcal{T}_{j}$.

3. For each edge of $\mathcal{T}_{j}$

- which belongs to the boundary $\delta \Omega$, join $Z_{j}$ to the middle point of the edge.

- which is common to a triangle $\mathcal{T}_{i}$, join $Z_{j}$ to $R_{i j}$.

Now we consider the space of piecewise quadratic $C^{1}$ continuous polynomials on $\Omega$, the PowellSabin splines. Each of the $6 t$ triangles resulting from the PS-refinement becomes the domain triangle of a quadratic Bernstein-Bézier polynomial (3.1) as indicated for one subtriangle in Figure 2a. Powell and Sabin [10] showed that the following interpolation problem:

$$
s\left(V_{k}\right)=f_{k}, \quad D_{x} s\left(V_{k}\right)=D_{x} f_{k}, \quad D_{y} s\left(V_{k}\right)=D_{y} f_{k}, \quad k=1, \ldots, n
$$


has a unique solution $s(x, y)$ in $S_{2}^{1}\left(\Delta_{P S}\right)$. Hence, the dimension of the space $S_{2}^{1}\left(\Delta_{P S}\right)$ equals $3 n$.

Dierckx [3] presented a normalized B-spline representation for Powell-Sabin splines

$$
s(x, y)=\sum_{i=1}^{n} \sum_{j=1}^{3} c_{i j} B_{i j}(x, y) \quad, \quad(x, y) \in \Omega
$$

where the B-splines form a convex partition of unity on $\Omega$, i.e.

$$
\begin{gathered}
B_{i j}(x, y) \geq 0 \text { for all } x, y \in \Omega, \\
\sum_{i=1}^{n} \sum_{j=1}^{3} B_{i j}(x, y)=1 \text { for all } x, y \in \Omega .
\end{gathered}
$$

Furthermore these basis functions have local support: $B_{i j}(x, y)$ vanishes outside the so-called molecule $M_{i}$ of vertex $V_{i}$, which is the union of all triangles $\mathcal{T}_{k}$ containing $V_{i}$.

The basis functions $B_{i j}(x, y)$ can be obtained as follows: find three linearly independent triplets $\left(\alpha_{i j}, \beta_{i j}, \gamma_{i j}\right), j=1,2,3$ for each vertex $V_{i}$. $B_{i j}(x, y)$ is the unique solution of the interpolation problem (3.3) with $\left(f_{k}, f_{x k}, f_{y k}\right)=\left(\delta_{k i} \alpha_{i j}, \delta_{k i} \beta_{i j}, \delta_{k i} \gamma_{i j}\right)$, where $\delta_{k i}$ is the Kronecker delta.

The triplets $\left(\alpha_{i j}, \beta_{i j}, \gamma_{i j}\right), j=1,2,3$, must be determined in such a way that equations (3.5) and (3.6) are satisfied. To find appropriate triplets $\left(\alpha_{i j}, \beta_{i j}, \gamma_{i j}\right), j=1,2,3$, we use the algorithm from $[3]$.

1. For each vertex $V_{i}$, find its PS-triangle points. These are the immediately surrounding Bézier domain points of the vertex $V_{i}$ and vertex $V_{i}$ itself. Figure 3 shows the PS-triangle points $L, \tilde{L}, L^{\prime}$ and $V_{1}$ for the vertex $V_{1}$ in the triangle $\mathcal{T}\left(V_{1}, V_{2}, V_{3}\right)$.

2. For each vertex $V_{i}$, find a triangle $t_{i}\left(Q_{i 1}, Q_{i 2}, Q_{i 3}\right)$ which contains all the PS-triangle points of $V_{i}$ from all the triangles $\mathcal{T}_{k}$ in the molecule $M_{i}$. These triangles $t_{i}, i=1, \ldots, n$ are called PS-triangles and we denote their vertices with $Q_{i j}\left(X_{i j}, Y_{i j}\right)$. Figure 3 also shows such a PS-triangle $t_{1}$.

3. Three linearly independent triplets of real numbers $\left(\alpha_{i j}, \beta_{i j}, \gamma_{i j}\right), j=1,2,3$ can be derived from the PS-triangle $t_{i}$ of a vertex $V_{i}$ as follows:

$\left(\alpha_{i 1}, \alpha_{i 2}, \alpha_{i 3}\right)$ are the barycentric coordinates of $V_{i}$ with respect to $t_{i}$,

$$
\begin{gathered}
\left(\beta_{i 1}, \beta_{i 2}, \beta_{i 3}\right)=\left(\frac{Y_{i 2}-Y_{i 3}}{h}, \frac{Y_{i 3}-Y_{i 1}}{h}, \frac{Y_{i 1}-Y_{i 2}}{h}\right), \\
\left(\gamma_{i 1}, \gamma_{i 2}, \gamma_{i 3}\right)=\left(\frac{X_{i 3}-X_{i 2}}{h}, \frac{X_{i 1}-X_{i 3}}{h}, \frac{X_{i 2}-X_{i 1}}{h}\right),
\end{gathered}
$$

where

$$
h=\left|\begin{array}{ccc}
X_{i 1} & Y_{i 1} & 1 \\
X_{i 2} & Y_{i 2} & 1 \\
X_{i 3} & Y_{i 3} & 1
\end{array}\right| .
$$

This allows to define the useful notion of control triangles. First, we define with the notation introduced above the PS-control points as

$$
C_{i j}\left(X_{i j}, Y_{i j}, c_{i j}\right) .
$$

For fixed $i$, they constitute a triangle $T_{i}\left(C_{i 1}, C_{i 2}, C_{i 3}\right)$ that is tangent to the surface at $\left(V_{i}, s\left(V_{i}\right)\right)$, see Figure 2b. The projection of the control triangles $T_{i}$ in the $(x, y)$ plane are the PS-triangles $t_{i}$. It is easily verified that

$$
A_{t_{i}}:=\operatorname{Area}\left(t_{i}\left(Q_{i 1}, Q_{i 2}, Q_{i 3}\right)\right)=\frac{1}{2\left|\beta_{i 1} \gamma_{i 2}-\gamma_{i 1} \beta_{i 2}\right|}=\frac{|f|}{2}
$$




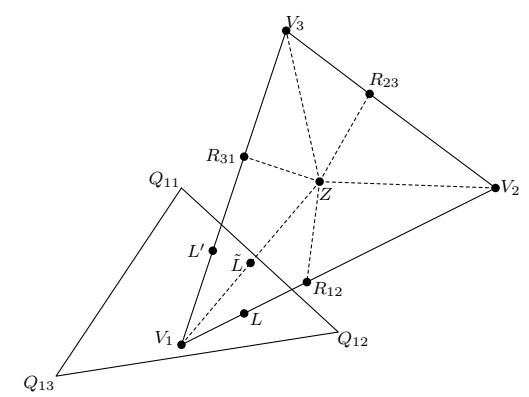

Figure 3: PS-triangle points and PS-triangle.

Obviously there are infinitely many possibilities for a PS-triangle because the only condition is that it contains the appropriate PS-triangle points. It is important to choose small PS-triangles in the construction of the basis functions. The control points will be closer to the surface, which gives the user more local control, and the stability of the basis will be better. Therefore Definition 3.1 introduces a constant $K$ that reflects the influence of the size of the PS-triangles. One can always find PS-triangles that satisfy $K=1$, and in practice $K$ will be typically smaller than 1 . If PS-triangles with minimal area are used, then $K$ can be bounded in function of the smallest angle in the triangulation. For more information we refer to [9].

Definition 3.1. Let $D_{i}$ be the smallest disk with vertex $V_{i}$ as center that contains all the PStriangle points of $V_{i}$ as in Figure 4 and denote its radius as $r_{i}$. An equilateral triangle $t_{D_{i}}$ with barycenter $V_{i}$ and inradius $K_{i} r_{i}$ with $K_{i} \geq 1$ obviously is a valid PS-triangle for $V_{i}$. We choose the value $K_{i}$ such that this equilateral triangle contains the actual PS-triangle $t_{i}$. Define $K=\max _{i} K_{i}$ as the maximum of all constants $K_{i}$ in the vertices $V_{i}$ of $\Delta$.

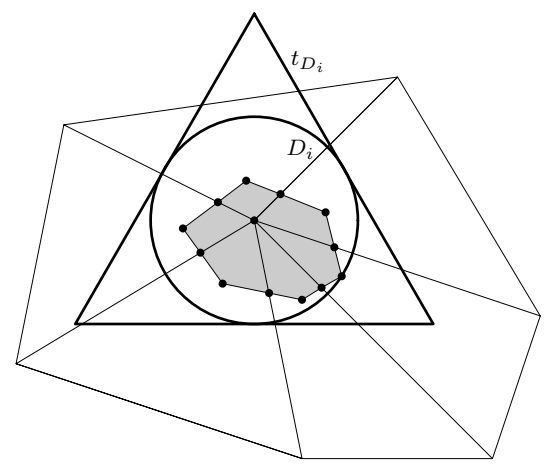

Figure 4: The disk $D_{i}$ and an equilateral triangle $t_{D_{i}}$ for $K=1$.

We recall that the normalized B-spline basis is a stable basis. The set $\left\{B_{i j}(x, y)\right\}$ is a stable basis if it satisfies (2.5). The following theorem gives explicit expressions for the constants $k_{1}$ and $k_{2}$ in $(2.5)$.

Theorem 3.2. Consider a triangulation $\Delta$ of a subset $\Omega \in \mathbb{R}^{2}$ with polygonal boundary $\delta \Omega$. Suppose $\Delta$ is constituted of triangles $\mathcal{T}_{j}, j=1, \ldots, t$, which have vertices $V_{i}, i=1, \ldots, n$. Define $\Delta_{P S}$ as the PS-refinement of $\Delta$. Then there exists a constant $k_{1}$ such that for all Powell-Sabin splines $s(x, y) \in S_{2}^{1}\left(\Delta_{P S}\right)$ in their normalized B-spline representation (3.4),

$$
k_{1}\|\mathbf{c}\|_{\infty} \leq\|s(x, y)\|_{\infty} \leq\|\mathbf{c}\|_{\infty}
$$


with $\|\mathbf{c}\|_{\infty}:=\max _{i j}\left|c_{i j}\right|,\|f\|_{\infty}:=\max _{\Omega}|f(x, y)|$ and

$$
k_{1}=\left[1+\frac{384 \sqrt{3} K}{\sin \left(\theta_{\Delta} \sin \left(\theta_{\Delta}\right) / 4\right)^{4} \tan \left(\theta_{\Delta} \sin \left(\theta_{\Delta}\right) / 8\right)}\right]^{-1} .
$$

Here $\theta_{\Delta}$ is the smallest angle in the triangulation $\Delta$ and $K$ is the constant defined in Definition 3.1 .

Proof. For the proof we refer to the work of Maes et al. [9].

\section{Approximation power of Powell-Sabin B-splines}

The main result of this section is Theorem 4.6 which states the existence of a linear quasiinterpolation operator $Q$ which maps $C(\Omega)$ onto the spline space $S_{2}^{1}\left(\Delta_{P S}\right)$ in such a way that if $f$ lies in a Sobolev space $W_{p}^{k+1}(\Omega)$ with $1 \leq k \leq 2$ and $2 \leq p \leq \infty$, then $Q f$ approximates $f$ and its derivatives to optimal order.

First we introduce some notations that will be used in this section, and we collect some properties of triangulations and Powell-Sabin refinements. Suppose $\Delta$ is a triangulation of a subset $\Omega \in \mathbb{R}^{2}$ with a PS-refinement $\Delta_{P S}$, and $\mathcal{T}$ is a triangle. With $|\mathcal{T}|$ we mean the diameter of the smallest disk containing $\mathcal{T}$, and $\rho_{\mathcal{T}}$ will denote the radius of the largest disk contained in $\mathcal{T}$. Denote the longest edge of $\mathcal{T}$ with $e_{\max }(\mathcal{T})$. The notations $\theta_{\mathcal{T}}, \theta_{\Delta}$ and $\theta_{\Delta_{P S}}$ will be used for the smallest angles in resp. $\mathcal{T}, \Delta$, and $\Delta_{P S}$. We give some estimates for the introduced quantities.

Property 4.1.

$$
\frac{|\mathcal{T}|}{\rho_{\mathcal{T}}} \leq \frac{4}{\tan \left(\theta_{\mathcal{T}} / 2\right)}
$$

Proof. It is well-known that

$$
\rho_{\mathcal{T}}=\tan \left(\theta_{\mathcal{T}} / 2\right) \cdot \frac{a+b-c}{2},
$$

with $a, b$ and $c$ the side lengths of the triangle. Side length $c$ corresponds to the side opposite to the angle $\theta_{\mathcal{T}}$, and thus has the smallest value. The following inequalities hold:

$$
\frac{2}{\tan \left(\theta_{\mathcal{T}} / 2\right)}=\frac{a+b-c}{\rho_{\mathcal{T}}} \geq \frac{\left|e_{\max }(\mathcal{T})\right|}{\rho_{\mathcal{T}}} \geq \frac{|\mathcal{T}| / 2}{\rho_{\mathcal{T}}} .
$$

Property 4.2.

$$
\theta_{\Delta_{P S}} \geq \theta_{\Delta} \sin \left(\theta_{\Delta}\right) / 4
$$

Proof. We refer to the work of Lai and Schumaker [7].

We will consider complex valued functions on a bounded domain $R$ in the 2 dimensional Euclidean space $\mathbb{R}^{2}$. Let $\alpha$ and $\beta$ denote positive integers. We define $L_{p}(R)$ as the set of functions $f$ such that $\int_{R}|f(x)|^{p} d x$ exists and is finite. For the case $p=\infty$ we define $L_{\infty}(R)$ as the set of functions $f$ such that $\max _{(x, y) \in R}|f(x, y)|<\infty$. The norm on $L_{p}(R)$ is given by $\|f\|_{p, R}=\left(\int_{R}|f(x)|^{p}\right)^{1 / p}$ and the norm on $L_{\infty}(R)$ by $\|f\|_{\infty, R}=\max _{(x, y) \in R}|f(x, y)|$. By $W_{p}^{k}(R)$ we mean the usual Sobolev space, i.e. the set of all functions in $L_{p}(R)$ whose distributional derivatives of order less than or equal to $k$ are in $L_{p}(R)$. The norm on $W_{p}^{k}(R)$ is $\|f\|_{k, p, R}=\sum_{\alpha+\beta \leq k}\left\|D_{x}^{\alpha} D_{y}^{\beta} f\right\|_{p, R}$. We will also use the semi-norm $|f|_{k, p, R}=\sum_{\alpha+\beta=k}\left\|D_{x}^{\alpha} D_{y}^{\beta} f\right\|_{p, R}$. For an excellent survey concerning Sobolev spaces the reader is referred to [1].

Before we prove the main theorem, we introduce three helpful lemmas. The first lemma bounds the size of the PS-triangles in function of the constant $K$ defined before. The second lemma is a form of Markov inequality for polynomials in $\mathcal{P}_{2}$ (3.1). The third lemma is the well-known Bramble-Hilbert lemma [2] which we state without proof for the specific case of functions in $\mathbb{R}^{2}$. 
Lemma 4.3. Denote the PS-triangle point with the longest distance to vertex $V_{i}$ as $S$ and define $\mathcal{T}_{S} \in \Delta_{P S}$ as either one of the two triangles that contains the PS-triangle point $S$. Then

$$
\frac{\left|e_{\max }\left(t_{i}\right)\right|}{\left|e_{\max }\left(\mathcal{T}_{S}\right)\right|} \leq \sqrt{3} K
$$

with $K$ the constant introduced in Definition 3.1 and $t_{i}$ the PS-triangle in vertex $V_{i}$.

Proof. By the definition of $K$ there exists an equilateral triangle $t_{D_{i}}$ that contains the PS-triangle $t_{i}$. Hence it is sufficient to prove that

$$
\frac{\left|e_{\max }\left(t_{D_{i}}\right)\right|}{\left|e_{\max }\left(\mathcal{T}_{S}\right)\right|} \leq \sqrt{3} K
$$

Denote $r_{i}$ as the radius of the disk $D_{i}$, defined in Definition 3.1. Then

$$
\left|e_{\max }\left(t_{D_{i}}\right)\right|=2 \sqrt{3} K_{i} r_{i} \leq 2 \sqrt{3} K r_{i} .
$$

If we combine this inequality with the fact that

$$
\left|e_{\max }\left(\mathcal{T}_{S}\right)\right| \geq 2\left|S V_{i}\right|=2 r_{i},
$$

then we have proven the lemma.

Lemma 4.4. Suppose $s(x, y) \in S_{2}^{1}\left(\Delta_{P S}\right)$ and let $1 \leq p \leq \infty$. Consider a triangle $\mathcal{T}_{P S}$ in the $P S$-refinement $\Delta_{P S}$ of $\Delta$. Then there exists a constant $C_{1}$ such that

$$
\left\|D_{x}^{\alpha} D_{y}^{\beta} s(x, y)\right\|_{p, \mathcal{T}_{P S}} \leq \frac{C_{1}}{\rho_{\mathcal{T}_{P S}}^{\alpha+\beta}}\|s(x, y)\|_{p, \mathcal{T}_{P S}}, \quad 0 \leq \alpha+\beta \leq 2,
$$

with $\rho_{\mathcal{T}_{P S}}$ the radius of the largest disk contained in $\mathcal{T}_{P S}$.

Proof. This follows immediately from Lemma 4.2 in [6].

Lemma 4.5 (Bramble-Hilbert [2]). Let $R$ be a bounded domain in $\mathbb{R}^{2}$ with diameter $|R|$, let $f$ be a function in $W_{p}^{k}(R)$, and let $F$ be a linear functional on $C^{j}(R)$ such that $0 \leq j<k$ satisfying

1. $|F(f)| \leq G \sum_{l=0}^{j}|R|^{l}|f|_{l, R}$ where $G$ is independent of $|R|$ and $f$ and

$$
|f|_{l, R}=\sup _{(x, y) \in R} \sum_{\alpha+\beta=l}\left|D_{x}^{\alpha} D_{y}^{\beta} f(x, y)\right|,
$$

2. $F(q)=0$ for all $q \in\left\{p(x, y) \mid D_{x}^{\alpha} D_{y}^{\beta} p(x, y)=0, \alpha+\beta=k\right\}$.

Then for $p>2 /(k-j)$ there is a constant $G_{1}$ independent of $|R|$ and $f$ such that

$$
|F(f)| \leq G_{1}|R|^{k-2 / p}|f|_{k, p, R}
$$

We will now prove that the best order of approximation by normalized Powell-Sabin B-splines is even achieved by a linear quasi-interpolation operator.

Theorem 4.6. Consider a triangulation $\Delta$ of a subset $\Omega \in \mathbb{R}^{2}$ with polygonal boundary $\delta \Omega$. Suppose $\Delta$ is constituted of triangles $\mathcal{T}_{j}, j=1, \ldots, t$, which have vertices $V_{i}, i=1, \ldots, n$. Define $\Delta_{P S}$ as the PS-refinement of $\Delta$. Fix $1 \leq k \leq 2$ and $2 \leq p \leq \infty$. Then there exists a linear quasiinterpolation operator $Q$ mapping $C(\Omega)$ onto $S_{2}^{1}\left(\Delta_{P S}\right)$ such that $Q f$ is in normalized B-spline representation (3.4) and for every $f \in W_{p}^{k+1}(\Omega)$,

$$
\left\|D_{x}^{\alpha} D_{y}^{\beta}(f-Q f)\right\|_{p, \Omega} \leq C|\Delta|^{k+1-\alpha-\beta}|f|_{k+1, p, \Omega},
$$

for all $0 \leq \alpha+\beta \leq k+1$. Here $|\Delta|$ is the maximum of the diameters of the triangles in $\Delta$ and $C$ is a constant. If $\alpha>0$ or $\beta>0$ then $C$ depends upon the smallest angle $\theta_{\Delta}$ in the triangulation $\Delta$. 
Proof. From (3.4) and the construction of the B-spline basis we have

$$
\left[\begin{array}{c}
s\left(V_{i}\right) \\
s_{x}\left(V_{i}\right) \\
s_{y}\left(V_{i}\right)
\end{array}\right]=A\left[\begin{array}{c}
c_{i 1} \\
c_{i 2} \\
c_{i 3}
\end{array}\right] \quad \text { with } \quad A=\left[\begin{array}{ccc}
\alpha_{i 1} & \alpha_{i 2} & \alpha_{i 3} \\
\beta_{i 1} & \beta_{i 2} & \beta_{i 3} \\
\gamma_{i 1} & \gamma_{i 2} & \gamma_{i 3}
\end{array}\right]
$$

This allows to define a quasi-interpolant $Q$ of the form

$$
Q f=\sum_{i=1}^{n} \sum_{j=1}^{3} \mu_{i j}(f) B_{i j}
$$

with

$$
\left[\begin{array}{c}
\mu_{i 1}(f) \\
\mu_{i 2}(f) \\
\mu_{i 3}(f)
\end{array}\right]:=A^{-1}\left[\begin{array}{c}
f\left(V_{i}\right) \\
f_{x}\left(V_{i}\right) \\
f_{y}\left(V_{i}\right)
\end{array}\right]
$$

Clearly the operator $Q$ satisfies

$$
\begin{gathered}
Q s=s, \quad \forall s \in S_{2}^{1}\left(\Delta_{P S}\right), \\
Q f\left(V_{i}\right)=f\left(V_{i}\right), \quad i=1, \ldots, n, \\
\nabla Q f\left(V_{i}\right)=\nabla f\left(V_{i}\right), \quad i=1, \ldots, n .
\end{gathered}
$$

If we take into account that $\alpha_{i 3}=1-\alpha_{i 1}-\alpha_{i 2}, \beta_{i 3}=-\beta_{i 1}-\beta_{i 2}$ and $\gamma_{i 3}=-\gamma_{i 1}-\gamma_{i 2}$, then we find that the inverse of $A$ is equal to

$$
A^{-1}=\left[\begin{array}{ccc}
1 & \eta_{i 1} & \tilde{\eta}_{i 1} \\
1 & \eta_{i 2} & \tilde{\eta}_{i 2} \\
1 & \eta_{i 3} & \tilde{\eta}_{i 3}
\end{array}\right]
$$

with

$$
\begin{aligned}
\eta_{i j} & :=\frac{\alpha_{i 2} \gamma_{i 1}-\alpha_{i 1} \gamma_{i 2}+\delta_{j 1} \gamma_{i 2}-\delta_{j 2} \gamma_{i 1}}{\beta_{i 1} \gamma_{i 2}-\beta_{i 2} \gamma_{i 1}} \\
\tilde{\eta}_{i j} & :=\frac{\alpha_{i 1} \beta_{i 2}-\alpha_{i 2} \beta_{i 1}-\delta_{j 1} \beta_{i 2}+\delta_{j 2} \beta_{i 1}}{\beta_{i 1} \gamma_{i 2}-\beta_{i 2} \gamma_{i 1}} .
\end{aligned}
$$

Fix $(x, y)$ in a triangle $\mathcal{T}_{P S} \in \Delta_{P S}$ with $\mathcal{T}_{P S}$ a subtriangle of triangle $\mathcal{T} \in \Delta$. By the stability of the normalized B-spline basis for $S_{2}^{1}\left(\Delta_{P S}\right)$,

$$
|Q f(x, y)| \leq \sum_{i \mid V_{i} \in \mathcal{T}} \sum_{j=1}^{3}\left|\mu_{i j}(f)\right| B_{i j}(x, y) \leq \max _{i \mid V_{i} \in \mathcal{T}, j}\left|f\left(V_{i}\right)+\eta_{i j} f_{x}\left(V_{i}\right)+\tilde{\eta}_{i j} f_{y}\left(V_{i}\right)\right| .
$$

We can find upper bounds for $\eta_{i j}$ and $\tilde{\eta}_{i j}$ by using the fact that $\left|\alpha_{i j}\right| \leq 1(3.7)$ and $\left|\delta_{i j}\right| \leq 1$, and by using the explicit formulas for $\beta_{i j}$ and $\gamma_{i j}$ given in (3.8) and (3.9)

$$
\left|\eta_{i j}\right| \leq\left|\alpha_{i 2} \gamma_{i 1}-\alpha_{i 1} \gamma_{i 2}+\delta_{j 1} \gamma_{i 2}-\delta_{j 2} \gamma_{i 1}\right| 2 A_{t_{i}} \leq 2\left(\left|X_{i 3}-X_{i 2}\right|+\left|X_{i 1}-X_{i 3}\right|\right) \leq 4\left|e_{\max }\left(t_{i}\right)\right| .
$$

Similarly we find that $\tilde{\eta}_{i j} \leq 4\left|e_{\max }\left(t_{i}\right)\right|$, and in combination with Lemma 4.3 we find

$$
\begin{aligned}
&\left|\eta_{i j}\right| \leq 4 \sqrt{3} K\left|e_{\max }\left(\mathcal{T}_{S}\right)\right| \leq 4 \sqrt{3} K|\mathcal{T}| \\
&\left|\tilde{\eta}_{i j}\right| \leq 4 \sqrt{3} K\left|e_{\max }\left(\mathcal{T}_{S}\right)\right| \leq 4 \sqrt{3} K|\mathcal{T}|
\end{aligned}
$$

with $|\mathcal{T}|$ the diameter of triangle $\mathcal{T}$ and $\mathcal{T}_{S}$ the subtriangle of $\mathcal{T}$ as defined in Lemma 4.3. By substituting the upper bounds for $\eta_{i j}$ and $\tilde{\eta}_{i j}$ in equation (4.1) we find that

$$
|Q f(x, y)| \leq\|f\|_{\infty, \mathcal{T}}+4 \sqrt{3} K|\mathcal{T}| \sup _{(x, y) \in \mathcal{T}}\left(\left|f_{x}(x, y)\right|+\left|f_{y}(x, y)\right|\right) .
$$


This immediately implies the existence of a constant $C_{2}$ such that

$$
|Q f(x, y)-f(x, y)| \leq C_{2} \sum_{l=0}^{1}|\mathcal{T}|^{l}|f|_{l, \mathcal{T}},
$$

with $|f|_{l, \mathcal{T}}$ defined as in Lemma 4.5. The Bramble-Hilbert lemma implies

$$
|Q f(x, y)-f(x, y)| \leq C_{3}|\mathcal{T}|^{k+1-2 / p}|f|_{k+1, p, \mathcal{T}}
$$

We find that

$$
\begin{aligned}
\|Q f(x, y)-f(x, y)\|_{p, \mathcal{T}} & =\left(\int_{\mathcal{T}}|Q f(x, y)-f(x, y)|^{p} d x d y\right)^{1 / p} \\
& \leq C_{3}|\mathcal{T}|^{k+1-2 / p}|f|_{k+1, p, \mathcal{T}} \cdot(\operatorname{Area}(\mathcal{T}))^{1 / p} \\
& \leq C_{3}|\mathcal{T}|^{k+1-2 / p}|f|_{k+1, p, \mathcal{T}} \cdot\left(\pi|\mathcal{T}|^{2}\right)^{1 / p} \\
& =C_{4}|\mathcal{T}|^{k+1}|f|_{k+1, p, \mathcal{T}} .
\end{aligned}
$$

This establishes the theorem for $\alpha=\beta=0$ and $p=\infty$. For $2 \leq p<\infty$ we have that

$$
\begin{aligned}
\|Q f(x, y)-f(x, y)\|_{p, \Omega} & =\left(\sum_{\mathcal{T} \in \Omega}\|Q f(x, y)-f(x, y)\|_{p, \mathcal{T}}^{p}\right)^{1 / p} \\
& \leq C_{4}|\Delta|^{k+1}\left(\sum_{\mathcal{T} \in \Omega}|f|_{k+1, p, \mathcal{T}}^{p}\right)^{1 / p} \\
& =C_{4}|\Delta|^{k+1}\left(\sum_{\mathcal{T} \in \Omega}\left(\sum_{\alpha+\beta=k+1}\left\|D_{x}^{\alpha} D_{y}^{\beta} f\right\|_{p, \mathcal{T}}\right)^{p}\right)^{1 / p} \\
& \leq C_{4}|\Delta|^{k+1}(k+2)\left(\sum_{\mathcal{T} \in \Omega} \max _{\alpha, \beta \mid \alpha+\beta=k+1}\left\|D_{x}^{\alpha} D_{y}^{\beta} f\right\|_{p, \mathcal{T}}^{p}\right)^{1 / p} \\
& \leq C_{4}|\Delta|^{k+1} 4\left(\sum_{\mathcal{T} \in \Omega} \sum_{\alpha+\beta=k+1}\left\|D_{x}^{\alpha} D_{y}^{\beta} f\right\|_{p, \mathcal{T}}^{p}\right)^{1 / p} \\
& =C_{5}|\Delta|^{k+1}|f|_{k+1, p, \Omega} .
\end{aligned}
$$

These equations establish the theorem for $\alpha=\beta=0$ and for arbitrary $p \geq 2$.

Fix $0 \leq \alpha+\beta \leq k+1$. Applying Lemma 4.4 to the polynomial $(Q f)||_{\mathcal{T}_{P S}}$ gives

$$
\left\|D_{x}^{\alpha} D_{y}^{\beta} Q f\right\|_{p, \mathcal{T}_{P S}} \leq \frac{C_{1}}{\rho_{\mathcal{T}_{P S}}^{\alpha+\beta}}\|Q f\|_{p, \mathcal{T}_{P S}} .
$$

Let $e$ and $\tilde{e}$ be two edges of an arbitrary subtriangle of $\mathcal{T}$. Then

$$
\sin \left(\theta_{\Delta_{P S}}\right)|e| \leq|\tilde{e}| .
$$

If we want to compare two arbitrary edges $e_{1}$ and $e_{2}$ of two arbitrary subtriangles of triangle $\mathcal{T} \in \Delta$, then there always exists a series of maximum five edges from $e_{1}$ to $e_{2}$ such that each pair of successive edges in the series has a common subtriangle in $\mathcal{T}$, hence

$$
\left|e_{1}\right| \leq\left(\frac{1}{\sin \left(\theta_{\Delta_{P S}}\right)}\right)^{4}\left|e_{2}\right| .
$$


If we apply this to (4.2), we find that

$$
\begin{aligned}
\left|\eta_{i j}\right| & \leq \frac{4 \sqrt{3} K}{\sin \left(\theta_{\Delta_{P S}}\right)^{4}}\left|\mathcal{T}_{P S}\right| \\
\left|\tilde{\eta}_{i j}\right| & \leq \frac{4 \sqrt{3} K}{\sin \left(\theta_{\Delta_{P S}}\right)^{4}}\left|\mathcal{T}_{P S}\right|
\end{aligned}
$$

with $\left|\mathcal{T}_{P S}\right|$ the diameter of triangle $\mathcal{T}_{P S}$. Combining (4.1) and (4.3) with these upper bounds yields

$$
\left|D_{x}^{\alpha} D_{y}^{\beta} Q f(x, y)\right| \leq \frac{C_{1}}{\rho_{\mathcal{T}_{P S}}^{\alpha+\beta}}\left[\|f\|_{\infty, \mathcal{T}}+\frac{4 \sqrt{3} K}{\sin \left(\theta_{\Delta_{P S}}\right)^{4}}\left|\mathcal{T}_{P S}\right| \sup _{(x, y) \in \mathcal{T}}\left(\left|f_{x}(x, y)\right|+\left|f_{y}(x, y)\right|\right)\right],
$$

and we find that

$$
\left|D_{x}^{\alpha} D_{y}^{\beta}(Q f-f)(x, y)\right| \leq \frac{C_{6}}{\rho_{\mathcal{T}_{P S}}^{\alpha+\beta}} \sum_{l=0}^{1}\left|\mathcal{T}_{P S}\right|^{l}|f|_{l, \mathcal{T}} \leq \frac{C_{6}}{\rho_{\mathcal{T}_{P S}}^{\alpha+\beta}} \sum_{l=0}^{1}|\mathcal{T}|^{l}|f|_{l, \mathcal{T}},
$$

where $C_{6}$ depends on the smallest angle $\theta_{\Delta_{P S}}$ in $\Delta_{P S}$. The Bramble-Hilbert theorem implies that

$$
\left|D_{x}^{\alpha} D_{y}^{\beta}(Q f-f)(x, y)\right| \leq \frac{C_{7}}{\rho_{\mathcal{T}_{P S}}^{\alpha+\beta}}|\mathcal{T}|^{k+1-2 / p}|f|_{k+1, p, \mathcal{T}}
$$

From Property 4.1 we get that

$$
\frac{1}{\rho_{\mathcal{T}_{P S}}} \leq \frac{4}{\tan \left(\theta_{\Delta_{P S}} / 2\right)\left|\mathcal{T}_{P S}\right|}
$$

and it is an easy exercise to prove that there exists a constant $C_{8}$ which depends only on the smallest angle $\theta_{\Delta_{P S}}$ in $\Delta_{P S}$ such that

$$
\left|\mathcal{T}_{P S}\right| \geq C_{8}|\mathcal{T}|
$$

Equations (4.4), (4.5) and (4.6) and Property 4.2 together yield

$$
\left|D_{x}^{\alpha} D_{y}^{\beta}(Q f-f)(x, y)\right| \leq C_{9}|\mathcal{T}|^{k+1-\alpha-\beta-2 / p}|f|_{k+1, p, \mathcal{T}}
$$

with $C_{9}$ a constant dependent only on $\theta_{\Delta}$. An analogous derivation as above proves the theorem for $0 \leq \alpha+\beta \leq k+1$ and $2 \leq p \leq \infty$.

\section{Triadic refinement}

We discuss a triadic scheme for refining a given triangulation $\Delta$ and its associated PS-refinement $\Delta_{P S}$ to produce nested sequences

$$
\Delta^{0} \subset \Delta^{1} \subset \Delta^{2} \subset \ldots \subset \Delta^{l}
$$

and

$$
\Delta_{P S}^{0} \subset \Delta_{P S}^{1} \subset \Delta_{P S}^{2} \subset \ldots \subset \Delta_{P S}^{l} .
$$

In the next section we review a subdivision scheme to compute a representation (3.4) of a PowellSabin spline on a triadic refinement $\Delta^{j+1}$ of a triangulation $\Delta^{j}[12]$.

Algorithm 5.1. Let $\Delta_{P S}^{0}$ be the PS-refinement associated with a triangulation $\Delta^{0}$. For each triangle $\mathcal{T}$ in $\Delta^{0}$,

1. add a new vertex $V_{i j k}$ such that this new vertex coincides with the interior vertex $Z_{i j k}$ of the $P S$-refinement $\Delta_{P S}$, 
2. insert two new vertices on the edges each at one side of the $R_{i j}$ and connect these vertices such that they form a hexagon. These new vertices have to be chosen in such a way that the resulting hexagon contains the interior vertex $V_{i j k}$,

3. connect the six new vertices on the edges with the interior vertex $V_{i j k}$,

4. for each of the nine new triangles, a new interior point is determined on the line of the old $P S$-refinement $\Delta_{P S}^{0}$ that crosses the new triangle.

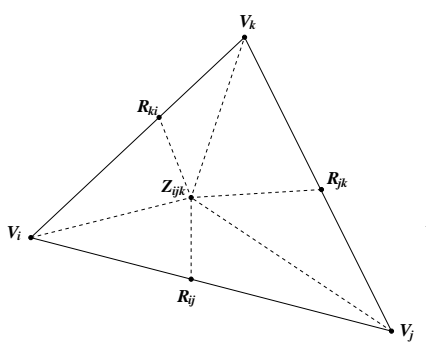

(a)

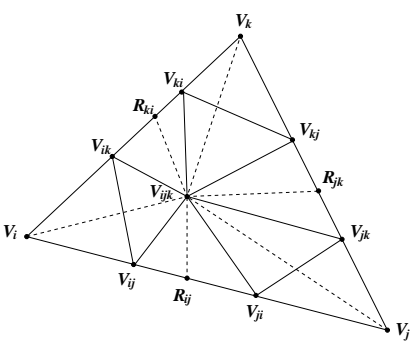

(b)

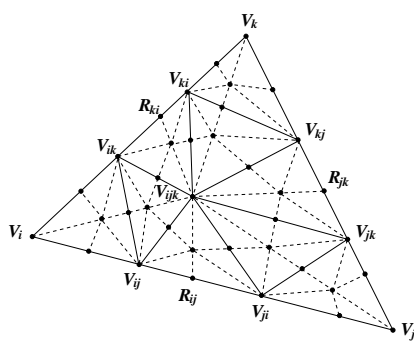

(c)

Figure 5: Principle of triadic subdivision. We place a new vertex at the position of the interior point $Z_{i j k}$ and two new vertices on the edges each at one side of the $R_{i j}$.

The steps of the algorithm are illustrated in Figure 5. It is clear that Algorithm 5.1 splits each triangle in $\Delta^{0}$ into nine subtriangles. This process can be repeated as often as desired to produce the nested sequences in (5.1) and (5.2).

Let $\mathcal{V}^{j}, \mathcal{E}^{j}$ and $\mathcal{T}^{j}$ denote the number of vertices, edges and triangles in the triangulation $\Delta^{j}$ obtained after $j$ steps of Algorithm 5.1 on an initial triangulation $\Delta^{0}$. Then the following equations can be deduced from Algorithm 5.1:

$$
\begin{aligned}
& \mathcal{T}^{j}=9 \mathcal{T}^{j-1} \\
& \mathcal{V}^{j}=\mathcal{V}^{j-1}+2 \mathcal{E}^{j-1}+\mathcal{T}^{j-1}
\end{aligned}
$$

and from Euler's formula we get that

$$
\mathcal{E}^{j}=\mathcal{V}^{j-1}+2 \mathcal{E}^{j-1}+10 \mathcal{T}^{j-1}-1
$$

Property 5.2. For all $j \geq 0$,

$$
\begin{aligned}
& \mathcal{T}^{j}=9^{j} \mathcal{T}^{0}, \\
& \mathcal{E}^{j}=3^{j-1} \mathcal{V}^{0}+2 \cdot 3^{j-1} \mathcal{E}^{0}-3^{j-1}+\left(\frac{3}{2} 9^{j}-\frac{7}{6} 3^{j}\right) \mathcal{T}^{0}, \\
& \mathcal{V}^{j}=3^{j-1} \mathcal{V}^{0}+2 \cdot 3^{j-1} \mathcal{E}^{0}-3^{j-1}+1+\left(\frac{9^{j}}{2}-\frac{7}{6} 3^{j}\right) \mathcal{T}^{0},
\end{aligned}
$$

Proof. This can easily be proved by induction.

In Table 1 we give the numbers $\mathcal{T}^{j}, \mathcal{E}^{j}$, and $\mathcal{V}^{j}$ for $j=0, \ldots, 8$, starting from two triangles with one common edge. For completion the table shows also the dimension of $S_{2}^{1}\left(\Delta_{P S}^{j}\right)$ which is equal to $3 \cdot \mathcal{V}^{j}$. 


\begin{tabular}{|r|r|r|r|r|}
\hline$j$ & $\mathcal{T}^{j}$ & $\mathcal{E}^{j}$ & $\mathcal{V}^{j}$ & $\operatorname{dim} S_{2}^{1}\left(\Delta_{P S}^{j}\right)$ \\
\hline 0 & 2 & 5 & 4 & 12 \\
1 & 18 & 33 & 16 & 48 \\
2 & 162 & 261 & 100 & 300 \\
3 & 1458 & 2241 & 784 & 2352 \\
4 & 13122 & 19845 & 6724 & 20172 \\
5 & 118098 & 177633 & 59536 & 178608 \\
6 & 1062882 & 1595781 & 532900 & 1598700 \\
7 & 9565938 & 14353281 & 4787344 & 14362032 \\
8 & 86093442 & 129153285 & 43059844 & 129179532 \\
\hline
\end{tabular}

Table 1: Number of triangles, edges, vertices and dimension in $S_{2}^{1}\left(\Delta_{P S}^{j}\right)$ for $j=0, \ldots, 8$.

\section{A hierarchical basis}

It is convenient to write the following in matrix form. Therefore we write a Powell-Sabin spline $s(x, y) \in S_{2}^{1}\left(\Delta_{P S}\right)$ as

$$
s(x, y)=\mathbf{\Phi c},
$$

where $\mathbf{\Phi}$ denotes the row vector of basis functions $B_{i j}(x, y)$ and $\mathbf{c}$ the column vector with the coefficients $c_{i j}$. A superscript $j$ denotes the resolution level as usual.

In [12], Vanraes et al. present a subdivision scheme to compute a representation (3.4) of a Powell-Sabin spline on a triadic refinement $\Delta^{j+1}$ of a triangulation $\Delta^{j}$. The result is a denser set of control points $\mathbf{c}^{j+1}$. The corresponding new basis functions $\boldsymbol{\Phi}^{j+1}$ have smaller support than the basis functions $\boldsymbol{\Phi}^{j}$ on the previous level and each of the functions in $\boldsymbol{\Phi}^{j}$ can be written as a linear combination of the functions in $\boldsymbol{\Phi}^{j+1}$, i.e. there exists a matrix $\mathbf{P}^{j}$ such that

$$
\boldsymbol{\Phi}^{j}=\boldsymbol{\Phi}^{j+1} \mathbf{P}^{j} .
$$

We obtain a multiresolution analysis, an increasing sequence of subspaces can be associated with the base triangulation $\Delta^{0}$. Vanraes et al. [12] give formula to compute the refined control points. The result can be written in block matrix form as

$$
\mathbf{c}^{j+1}=\left[\begin{array}{c}
\mathbf{O}^{j} \\
\mathbf{N}^{j}
\end{array}\right] \mathbf{c}^{j}
$$

The part $\mathbf{O}^{j}$ computes new control points for the old vertices that are contained in both $\Delta^{j}$ and $\Delta^{j+1}$, and the part $\mathbf{N}^{j}$ computes control points for the new vertices that are added when going from $\Delta^{j}$ to $\Delta^{j+1}$. We remark that $\mathbf{O}^{j}$ is invertible, the new control triangle for an old vertex is in fact only a rescaled version of the old control triangle. Furthermore the subdivsion formula are convex combinations, i.e. the rows of $\mathbf{O}^{j}$ and $\mathbf{N}^{j}$ contain a finite number of elements smaller than one and each row sums up to one. This means the subdivision algorithm is stable.

We split $\boldsymbol{\Phi}^{j+1}$ in functions $\mathcal{O}^{j+1}$ associated with the old vertices in $\Delta^{j}$ and functions $\mathcal{N}^{j+1}$ associated with the new vertices that are added when going from $\Delta^{j}$ to $\Delta^{j+1}$

$$
\boldsymbol{\Phi}^{j+1}=\left[\mathcal{O}^{j+1} \mathcal{N}^{j+1}\right] .
$$

Define the set of functions $\boldsymbol{B}^{m}(2.2)$ as

$$
\mathcal{B}^{m}:=\left\{\boldsymbol{\Phi}^{0} \cup \bigcup_{j=1}^{m} \mathcal{N}^{j}\right\}
$$

for each $m=0, \ldots, l$ and define the set $\mathcal{B}$ as in (2.1).

Theorem 6.1. For each $0 \leq m \leq l$, the set of splines $\mathcal{B}^{m}$ (6.1) forms a basis for $S_{2}^{1}\left(\Delta_{P S}^{m}\right)$. 
Proof. From the previous we know that

$$
\left[\begin{array}{ll}
\boldsymbol{\Phi}^{j} & \mathcal{N}^{j+1}
\end{array}\right]=\boldsymbol{\Phi}^{j+1}\left[\begin{array}{ll}
\mathbf{O}^{j} & \mathbf{0} \\
\mathbf{N}^{j} & \mathbf{1}
\end{array}\right] .
$$

Because $\mathbf{O}^{j}$ is invertible, the matrix $\left[\begin{array}{ll}\mathbf{O}^{j} & \mathbf{0} \\ \mathbf{N}^{j} & \mathbf{1}\end{array}\right]$ is also invertible, and we can write

$$
\left[\begin{array}{cc}
\left(\mathbf{O}^{j}\right)^{-1} & \mathbf{0} \\
-\mathbf{N}^{j}\left(\mathbf{O}^{j}\right)^{-1} & \mathbf{1}
\end{array}\right]\left[\begin{array}{ll}
\boldsymbol{\Phi}^{j} & \mathcal{N}^{j+1}
\end{array}\right]=\boldsymbol{\Phi}^{j+1} .
$$

Hence for all $j$ we have proven that the set of functions $\left[\begin{array}{ll}\boldsymbol{\Phi}^{j} & \mathcal{N}^{j+1}\end{array}\right]$ forms a basis for the space $V^{j+1}$. The theorem follows by induction.

Theorem 6.1 shows that $\mathcal{B}$ is a hierarchical basis for $S_{2}^{1}\left(\Delta_{P S}^{l}\right)$. Every spline $s(x, y) \in S_{2}^{1}\left(\Delta_{P S}^{l}\right)$ has a unique hierarchical representation

$$
s(x, y)=\sum_{j=0}^{l} \sum_{i=1}^{n_{j}} c_{i}^{j} \mathcal{B}_{i}^{j}(x, y),
$$

with $\mathcal{B}_{i}^{0}:=\boldsymbol{\Phi}_{i}^{0}$ and $\mathcal{B}_{i}^{j}:=\mathcal{N}_{i}^{j}, 1 \leq j \leq l$. The basis functions in Theorem 6.1 are local and stable. Define $M^{j}(V)$ as the molecule of vertex $V$ in triangulation $\Delta^{j}$, i.e. the union of all triangles $\mathcal{T}_{k} \in \Delta^{j}$ containing vertex $V$.

Theorem 6.2. For each $0 \leq j \leq l$, the supports and sizes of $\mathcal{B}_{i}^{j}$ satisfy

1. $\operatorname{supp} \mathcal{B}_{i}^{j} \subseteq M^{j}(V)$ if the coefficient $c_{i}^{j}$ is associated with vertex $V \in \Delta^{j}$,

2. $\mathcal{B}_{i}^{j}(x, y) \geq 0$,

3. $\sum_{i=1}^{n_{j}} \mathcal{B}_{i}^{j}(x, y) \leq 1$.

Proof. This follows immediately from the construction of the hierarchical basis and from properties (3.5) and (3.6).

We will now show that the spline $s(x, y)$ in its unique hierarchical representation (6.2) is stable in the sense that if $s(x, y)$ has small coefficients, then $\|s(x, y)\|_{\infty}$ is also small.

Theorem 6.3. Suppose $s(x, y) \in S_{2}^{1}\left(\Delta_{P S}^{l}\right)$ is a spline in its unique hierarchical representation (6.2) whose coefficients satisfy

$$
\left|c_{i}^{j}\right| \leq \frac{\epsilon}{l+1}
$$

Then $\|s(x, y)\|_{\infty} \leq \epsilon$.

Proof. Fix $(x, y) \in \Omega$, then

$$
\begin{aligned}
|s(x, y)| & \leq \sum_{j=0}^{l} \sum_{i=1}^{n_{j}}\left|c_{i}^{j}\right| \mathcal{B}_{i}^{j}(x, y) \\
& \leq \sum_{j=0}^{l} \frac{\epsilon}{l+1} \sum_{i=1}^{n_{j}} \mathcal{B}_{i}^{j}(x, y)
\end{aligned}
$$

The claim that $\|s(x, y)\|_{\infty} \leq \epsilon$ follows from property 3 in Theorem 6.2. 


\section{Compression}

Define $\mathcal{V}_{l}$ as the set of vertices in $\Delta^{l}$. Because of $(3.3)$, a spline $s(x, y) \in S_{2}^{1}\left(\Delta_{P S}^{l}\right)$ is uniquely determined by the values

$$
\bigcup_{V \in \mathcal{V}_{l}}\left\{s(V), s_{x}(V), s_{y}(V)\right\} .
$$

Define $Q^{m}$ as the linear quasi-interpolation operator mapping $C(\Omega)$ onto $S_{2}^{1}\left(\Delta_{P S}^{m}\right)$ as defined in Theorem $4.6, m=0, \ldots, l$. Define

$$
\hat{s}^{0}:=Q^{0} s(x, y)
$$

and

$$
\hat{s}^{m}:=Q^{m}\left(s(x, y)-\sum_{j=0}^{m-1} \hat{s}^{j}\right), \quad m=1, \ldots, l .
$$

We can write $\hat{s}^{m}$ in matrix form as

$$
\mathbf{\Phi}^{m} \mathbf{c}^{m}, \quad m=0, \ldots, l .
$$

As before, let $\boldsymbol{\Phi}^{m}=\left[\mathcal{O}^{m} \mathcal{N}^{m}\right]$ and analogously $\mathbf{c}^{m}=\left[\mathbf{c}_{o}^{m} \mathbf{c}_{n}^{m}\right]$. It is clear from the construction of $\hat{s}^{m}$ that the subset of coefficients $\mathbf{c}_{o}^{m}$ corresponding to the basisfunctions $\mathcal{O}^{m}$ are zero for $m=1, \ldots, l$. This construction can easily be turned into an algorithm for computing the coefficients in (6.2). We refer to this process of computing the coefficients in (6.2) from the values (7.1) as decomposition.

Algorithm 7.1 (Decomposition).

1. Use (7.2) to compute $\left\{c_{i}^{0}\right\}_{i=1}^{n_{0}} \equiv \mathbf{c}^{0}$ from (7.1).

2. For $m=1, \ldots, l$,

(a) use (7.3) to form the spline $\hat{s}^{m}$ from (7.1),

(b) compute $\left\{c_{i}^{m}\right\}_{i=1}^{n_{m}} \equiv \mathbf{c}_{n}^{m}$.

In view of Theorem 6.3 we can now describe a thresholding strategy.

\section{Algorithm 7.2 (Thresholding).}

1. Choose $\epsilon$.

2. For each $m=0, \ldots, l$, drop the coefficients in $\left\{c_{i}^{m}\right\}_{i=1}^{n_{m}}$ that are smaller than the threshold $\epsilon$.

Our decomposition algorithm will give good compression rates when the hierarchical expansion (6.2) contains many small coefficients. The following theorem guarantees that the coefficients become smaller with each hierarchic level $m$.

Theorem 7.3. Fix $1 \leq k \leq 2$ and $2 \leq p \leq \infty$. Given is a function $f \in W_{p}^{k+1}(\Omega)$. Suppose $s \in S_{2}^{1}\left(\Delta_{P S}^{l}\right)$ is the unique solution of the interpolation problem (3.3). Then for all $0 \leq m \leq l-1$,

$$
\left\|s-s_{m}\right\|_{p, \Omega} \leq C\left|\Delta^{m}\right|^{k+1}|f|_{k+1, p, \Omega} .
$$

Here $\left|\Delta^{m}\right|$ is the maximum of the diameters of the triangles in $\Delta^{m}$, and $C$ is a constant.

Proof. By Theorem 4.6 we have that

$$
\|s-f\|_{p, \Omega} \leq C_{1}\left|\Delta^{l}\right|^{k+1}|f|_{k+1, p, \Omega}
$$

and

$$
\left\|f-s_{m}\right\|_{p, \Omega} \leq C_{2}\left|\Delta^{m}\right|^{k+1}|f|_{k+1, p, \Omega}
$$

for all $m$. Then from the triangle inequality we find that

$$
\begin{aligned}
\left\|s-s_{m}\right\|_{p, \Omega} & \leq\|s-f\|_{p, \Omega}+\left\|f-s_{m}\right\|_{p, \Omega} \\
& \leq\left(C_{1}\left|\Delta^{l}\right|^{k+1}+C_{2}\left|\Delta^{m}\right|^{k+1}\right)|f|_{k+1, p, \Omega} \\
& \leq\left(C_{1}+C_{2}\right)\left|\Delta^{m}\right|^{k+1}|f|_{k+1, p, \Omega} .
\end{aligned}
$$

Setting $C=C_{1}+C_{2}$ proves the theorem. 
Suppose that $f \in W_{p}^{3}(\Omega)$. Then the theorem implies the following: if $s$ is the solution of the interpolation problem (3.3), then the coefficients at level $m$ will be approximately $1 / 27$ as large as the analogous coefficients at level $m-1$. Hence at higher levels many coefficients should be small and can be removed in the thresholding step.

\section{Numerical examples}

To demonstrate the performance of the compression algorithm, we performed experiments with several test functions. In all cases we choose $\Delta^{0}$ as the triangulation that is constructed by dividing the unit square $[0,1] \times[0,1]$ by its bissectrice in two triangles. We selected six bivariate test functions which are given by

$$
\begin{aligned}
f_{1}(x, y)= & 0.75 \exp \left[-\frac{(9 x-2)^{2}+(9 y-2)^{2}}{4}\right]+0.75 \exp \left[-\frac{(9 x+1)^{2}}{49}-\frac{(9 y+1)}{10}\right] \\
& +0.5 \exp \left[-\frac{(9 x-7)^{2}+(9 y-3)^{2}}{4}\right]-0.2 \exp \left[-(9 x-4)^{2}-(9 y-7)^{2}\right], \\
f_{2}(x, y)= & (\tanh (9-9 x-9 y)+1) / 9, \\
f_{3}(x, y)= & (1.25+\cos (5.4 y)) /\left(6+6(3 x-1)^{2}\right), \\
f_{4}(x, y)= & \exp \left[-\frac{81}{4}\left((x-0.5)^{2}+(y-0.5)^{2}\right)\right] / 3, \\
f_{5}(x, y)= & \sqrt{64-81\left((x-0.5)^{2}+(y-0.5)^{2}\right) / 9-0.5}, \\
f_{6}(x, y)= & \begin{cases}\exp \left[-\frac{r_{0}^{2}}{r_{0}^{2}-r^{2}}\right], & r<r_{0}, \\
0, & \text { otherwise },\end{cases} \\
& \text { with } r:=r(x, y)=(x-0.5)^{2}+(y-0.5)^{2} \text { and } r_{0}=1 / 128 .
\end{aligned}
$$

For each test function $f$ we measure the root mean square (RMS) error between $f$ and $s$. That is,

$$
R M S=\sqrt{\frac{\sum_{i=0}^{M} \sum_{j=0}^{N}\left(f\left(x_{i}, y_{j}\right)-s\left(x_{i}, y_{j}\right)\right)^{2}}{(M+1)(N+1)}},
$$

where $x_{i}=i / M, y_{j}=j / N$, and $M=N=100$. We also measure the maximum distance (MD) between $f$ and $s$,

$$
M D=\max _{i=0, \ldots, M, j=0, \ldots, N}\left|f\left(x_{i}, y_{j}\right)-s\left(x_{i}, y_{j}\right)\right| .
$$

To normalize the error values, we divide the RMS error and MD error by the difference of the maximum and minimum values of $f$ over the domain. These normalized error values are denoted by NRMS and NMD. Each test function $f_{i}$ is approximated by a spline $s_{l}$ corresponding to level $l=1$ or $l=2$, and by a spline $s_{4}$ which is compressed with threshold $\epsilon$. We choose the threshold $\epsilon$ such that the spline $s_{4}$ after compression with threshold $\epsilon$ has less non-zero coefficients than the spline $s_{l}$. The results are shown in Table 2 and Table 3 . The surfaces corresponding to the functions $f_{i}$ are plotted in Figure 6, the surfaces corresponding to the splines $s_{l}$ are plotted in Figure 7 and the surfaces corresponding to the compressed splines $s_{4}$ are plotted in Figure 8 .

\section{Application: image compression}

Our surface compression algorithm is well suited to any application that can be cast as a surface fitting problem. We can interpret a grayscale image as a surface, where the value of each pixel represents its height. Image compression is then cast into a surface compression framework that can be solved with the algorithm presented in this paper. Derivative information of the image surface can be estimated by applying the Sobel operator [8] to the image. 


\begin{tabular}{|c|c|c|c|c|c|c|}
\hline & $f_{1}$ & $f_{2}$ & $f_{3}$ & $f_{4}$ & $f_{5}$ & $f_{6}$ \\
\hline$l$ & 1 & 1 & 2 & 1 & 1 & 2 \\
\hline \# coeff & 48 & 48 & 300 & 48 & 48 & 300 \\
\hline RMS & 0.040139 & 0.012541 & 0.000158 & 0.020974 & 0.001675 & 0.034707 \\
\hline MD & 0.139814 & 0.035804 & 0.000989 & 0.103371 & 0.007246 & 0.353521 \\
\hline NRMS & 0.032954 & 0.056434 & 0.000432 & 0.062926 & 0.004783 & 0.094343 \\
\hline NMD & 0.114785 & 0.161119 & 0.002698 & 0.310126 & 0.020687 & 0.960970 \\
\hline
\end{tabular}

Table 2: Errors between the test functions $f_{i}(x, y)$ and the approximating spline $s_{l}(x, y)$ without compression.

\begin{tabular}{|c|c|c|c|c|c|c|}
\hline & $f_{1}$ & $f_{2}$ & $f_{3}$ & $f_{4}$ & $f_{5}$ & $f_{6}$ \\
\hline$\epsilon$ & 0.1 & 0.04 & 0.0008 & 0.02 & 0.015 & 0.01 \\
\hline \# coeff & 47 & 45 & 256 & 36 & 48 & 276 \\
\hline rate & 429 to 1 & 448 to 1 & 79 to 1 & 560 to 1 & 420 to 1 & 73 to 1 \\
\hline RMS & 0.030971 & 0.009209 & 0.000159 & 0.004838 & 0.001675 & 0.000772 \\
\hline MD & 0.108095 & 0.024047 & 0.000647 & 0.013934 & 0.007246 & 0.019024 \\
\hline NRMS & 0.025427 & 0.041442 & 0.000435 & 0.014514 & 0.004783 & 0.002098 \\
\hline NMD & 0.088745 & 0.108213 & 0.001765 & 0.041804 & 0.020687 & 0.051713 \\
\hline
\end{tabular}

Table 3: Errors between the test functions $f_{i}(x, y)$ and the approximating spline $s_{4}(x, y)$ after compression with threshold $\epsilon$.

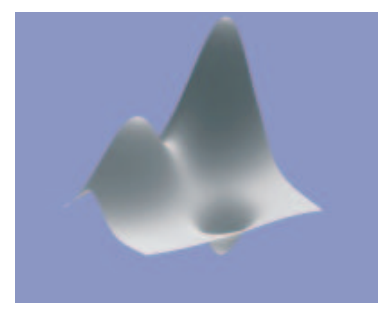

(a) $f_{1}$

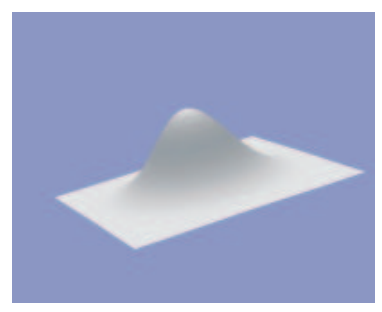

(d) $f_{4}$

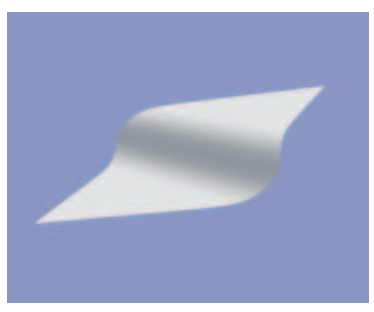

(b) $f_{2}$

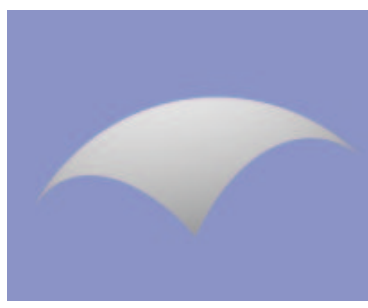

(e) $f_{5}$

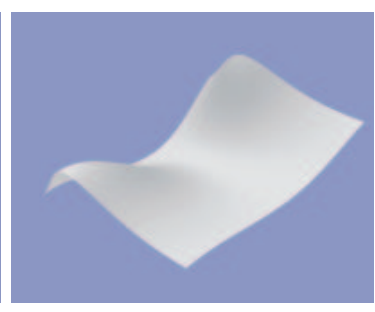

(c) $f_{3}$

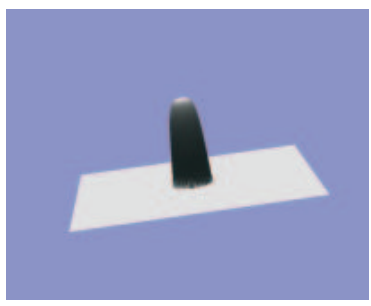

(f) $f_{6}$

Figure 6: The test functions. 


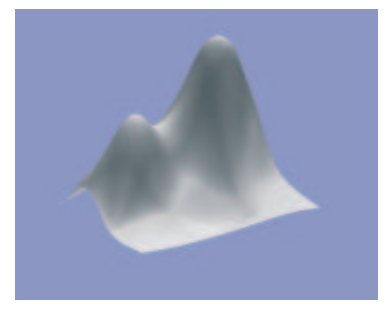

(a) $s_{1}$

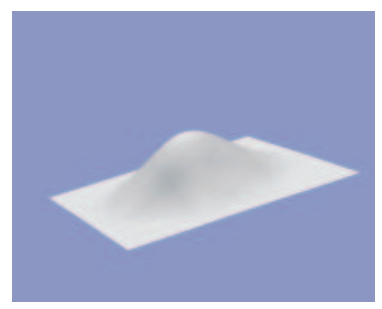

(d) $s_{1}$

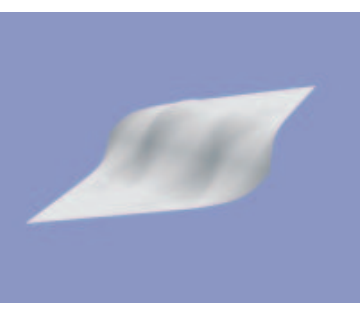

(b) $s_{1}$

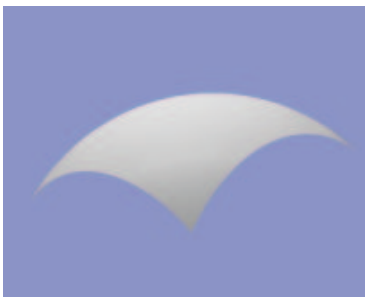

(e) $s_{1}$

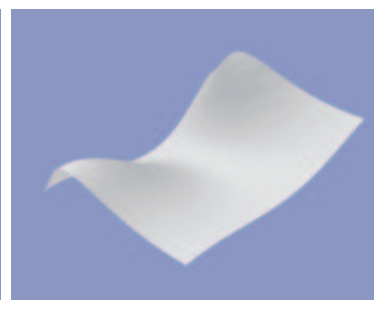

(c) $s_{2}$

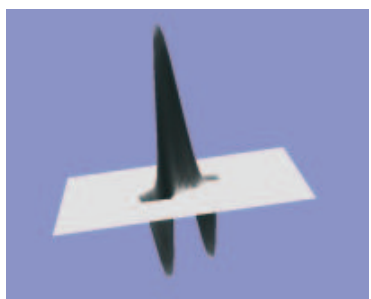

(f) $s_{2}$

Figure 7: The approximating splines $s_{l}$ at level $l=1$ or $l=2$.

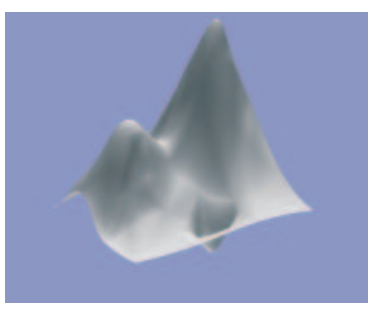

(a)

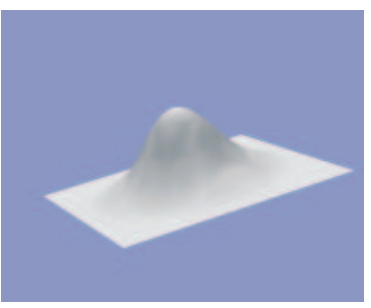

(d)

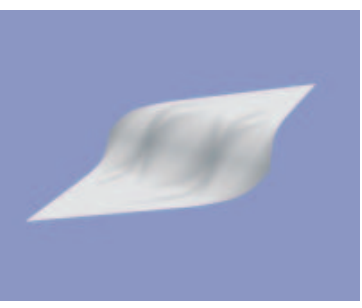

(b)

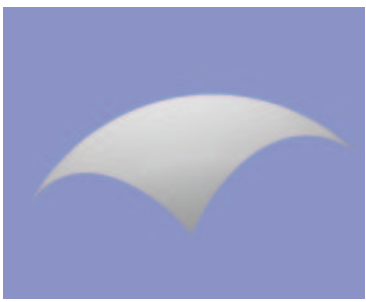

(e)

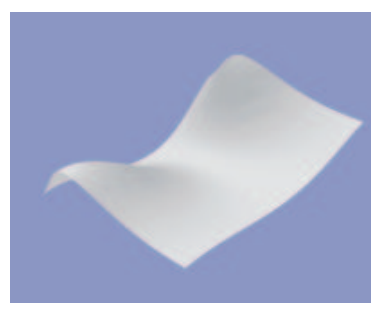

(c)

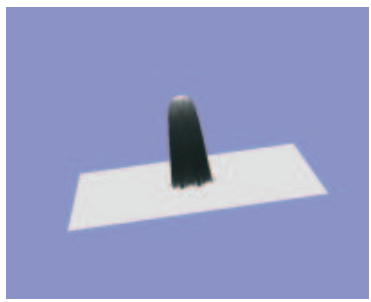

(f)

Figure 8: The splines $s_{4}$ after compression. 
The dimensions of the original image in Figure $9 \mathrm{a}$ are $512 \times 512$. We used a spline $s_{5}$ to approximate the original surface. The base domain $\Delta^{0}$ is constructed by dividing the unit square $[0,1] \times[0,1]$ by its bissectrice in two triangles. The compression algorithm was applied with threshold values $\epsilon$ equal to $0.01,0.02,0.03,0.04$, and 0.05 . The number of non-zero coefficients for the images in Figures $9 \mathrm{~b}-9 \mathrm{f}$ are respectively 79685, 45210, 31237, 22315, 17439. The corresponding compression rates are respectively 3.3 to $1,5.8$ to $1,8.4$ to $1,11.7$ to 1 , and 15.0 to 1 .

\section{Acknowledgements}

This work is partially supported by the Flemish Fund for Scientific Research (FWO Vlaanderen) project MISS (G.0211.02), and by the Belgian Programme on Interuniversity Attraction Poles, initiated by the Belgian Federal Science Policy Office. The scientific responsibility rests with the authors.

\section{References}

[1] R. A. Adams. Sobolev spaces. Academic Press, New York, 1975.

[2] J. H. Bramble and S. R. Hilbert. Bounds for a class of linear functionals with applications to hermite interpolation. Numer. Math., 16:362-369, 1971.

[3] P. Dierckx. On calculating normalized Powell-Sabin B-splines. Comput. Aided Geom. Design, 15(3):61-78, 1997.

[4] G. Farin. Triangular Bernstein-Bézier patches. Comput. Aided Geom. Design, 3(2):83-128, 1986 .

[5] D. Hong and L. L. Schumaker. Surface compression using a basis of $C^{1}$ cubic bivariate spline spaces. Journal of Computing, 2004. To appear.

[6] M. Lai and L. Schumaker. On the approximation power of bivariate splines. Advances in Comp. Math., 9:251-279, 1998.

[7] M. Lai and L. Schumaker. Macro-elements and stable local bases for splines on Powell-Sabin splits. Math. Comp., 72:335-354, 2003.

[8] J. S. Lim. Two Dimensional Signal and Image Processing. Prentice-Hall, International editions, 1990

[9] J. Maes, E. Vanraes, P. Dierckx, and A. Bultheel. On the stability of normalized Powell-Sabin B-splines. J. Comput. Appl. Math., 2004. To appear.

[10] M. J. D. Powell and M. A. Sabin. Piecewise quadratic approximations on triangles. ACM Transactions on Mathematical Software, 3:316-325, 1977.

[11] E. Vanraes, J. Maes, and A. Bultheel. Powell-Sabin spline wavelets. Intl. Journal of Wavelets, Multiresolution and Information Processing, 2(1):23-42, 2004.

[12] E. Vanraes, J. Windmolders, A. Bultheel, and P. Dierckx. Automatic construction of control triangles for subdivided Powell-Sabin splines. Computer Aided Geometric Design, 2004. Accepted. 


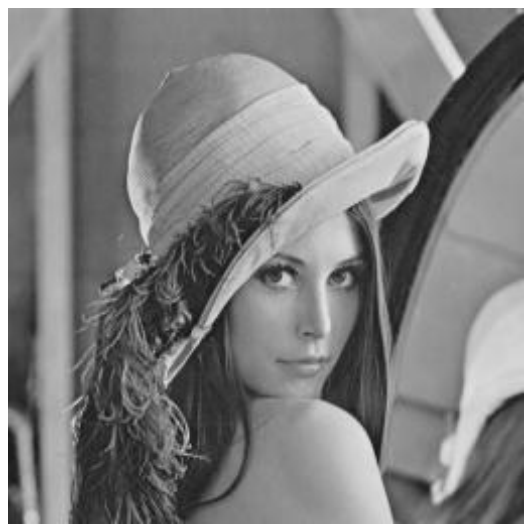

(a) original

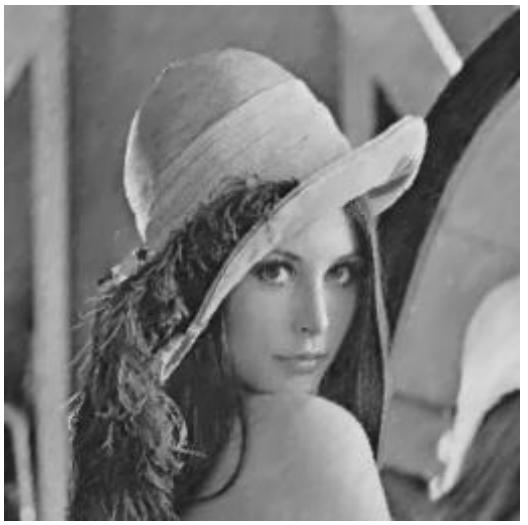

(c) 5.8 to 1

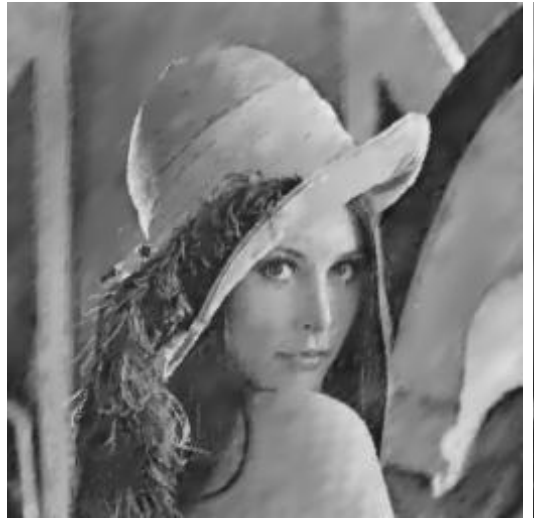

(e) 11.7 to 1

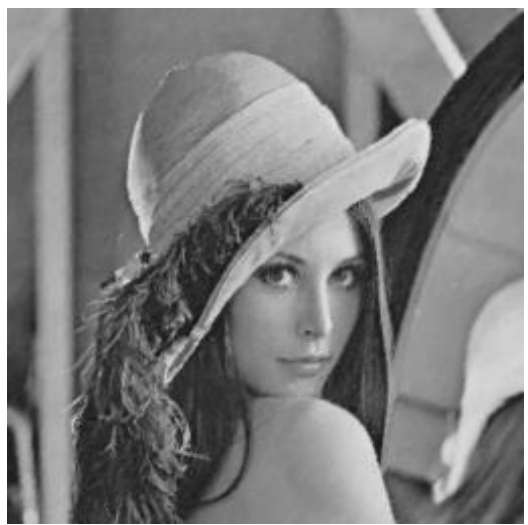

(b) 3.3 to 1

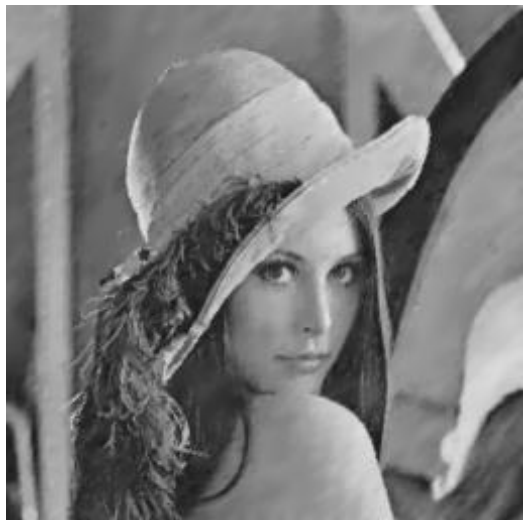

(d) 8.4 to 1

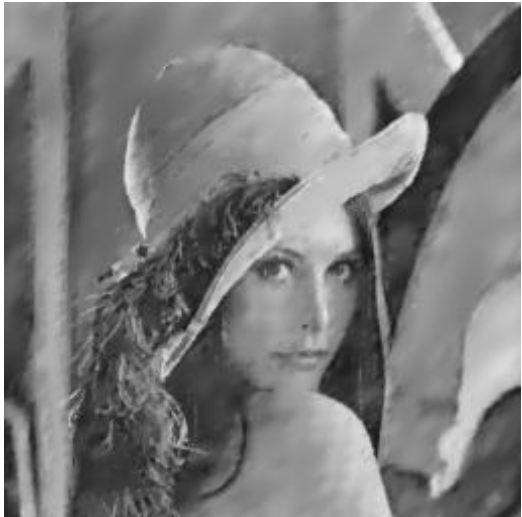

(f) 15.0 to 1

Figure 9: Image compression on a picture of Lena. 